\title{
MR Susceptibility Imaging for Detection of Tumor- Associated Macrophages in Glioblastoma
}

\section{Amir Nazem}

University of Pennsylvania

Samantha Guiry

UPenn

Mehrdad Pourfathi

Upenn

Jeffrey B. Ware

UPenn

Hannah Anderson

UPenn

Srikant Kamesh lyer

UPenn

Brianna F Moon

UPenn

Yi Fan

UPenn

Walter R. Witschey

UPenn

\section{Rahim Rizi}

UPenn

Stephen J. Bagley

UPenn

Arati Desai

UPenn

Donald M. O'Rourke

UPenn

Steven Brem

UPenn

MacLean Nasrallah

UPenn

Ali Nabavizadeh ( $\square$ ali.nabavizadeh@pennmedicine.upenn.edu )

Upenn: University of Pennsylvania https://orcid.org/0000-0002-0380-4552 


\section{Research Article}

Keywords: Tumor-associated macrophages, Glioblastoma, MRI, quantitative susceptibility mapping Posted Date: November 1st, 2021

DOI: https://doi.org/10.21203/rs.3.rs-1017881/v1

License: (c) (i) This work is licensed under a Creative Commons Attribution 4.0 International License. Read Full License

Version of Record: A version of this preprint was published at Journal of Neuro-Oncology on January 19th, 2022. See the published version at https://doi.org/10.1007/s11060-022-03947-3. 


\section{Abstract \\ Purpose}

Tumor-associated macrophages (TAMs) are a key component of glioblastoma (GBM) microenvironment. Considering the differential role of different TAM phenotypes in iron metabolism with the M1 phenotype storing intracellular iron, and M2 phenotype releasing iron in the tumor microenvironment, we investigated MRI to quantify iron as an imaging biomarker for TAMs in GBM patients.

\section{Methods}

21 adult patients with GBM underwent a 3D single echo gradient echo MRI sequence and quantitative susceptibility maps were generated. In 3 subjects, ex vivo imaging of surgical specimens was performed on a 9.4 Tesla MRI using 3D multi-echo GRE scans, and R2* $\left(1 / T 2^{\star}\right)$ maps were generated. Each specimen was stained with hematoxylin and eosin, as well as CD68, CD86, CD206, and L-Ferritin.

\section{Results}

Significant positive correlation was observed between mean susceptibility for the tumor enhancing zone and the L-ferritin positivity percent $(r=0.56, p=0.018)$ and the combination of tumor's enhancing zone and necrotic core and the L-Ferritin positivity percent $(r=0.72 ; p=0.001)$. The mean susceptibility significantly correlated with positivity percent for $\operatorname{CD} 68(\rho=0.52, p=0.034)$ and $\operatorname{CD} 86(r=0.7 p=0.001)$, but not for CD206 $(\rho=0.09 ; p=0.7)$. There was a positive correlation between mean $R 2^{*}$ values and CD68 positive cell counts $(r=0.6, p=0.016)$. Similarly, mean $R 2 *$ values significantly correlated with CD86 $(r=0.54$, $p=0.03)$ but not with CD206 $(r=0.15, p=0.5)$.

\section{Conclusion}

MR quantitative susceptibility mapping can quantify the iron content of GBM and provide a non-invasive method for TAM quantification and phenotyping.

\section{Introduction}

Glioblastoma (GBM) is the most common malignant primary brain tumor, with a median survival of 15 months, and a 2-year survival rate of $26.5 \%$ despite an aggressive treatment regimen including surgical resection and chemoradiotherapy (1). Several mechanisms have been proposed for treatment failure which include tumor heterogeneity and immunosuppressive tumor microenvironment (2).

Pathologically, GBM is characterized by a heterogeneous microenvironment consisting of not only neoplastic but also non-neoplastic cells including resident and migrated immune cells, vascular cells, as 
well as other glial cells (3). Among the non-neoplastic components of GBM microenvironment tumorassociated macrophages (TAMs) are the dominant immune cell population with a key role in tumor growth (4).

TAMs exert a pro-tumor behavior by suppressing the innate immune response, promoting tumor angiogenesis, as well as releasing the intracellular iron into the tumor microenvironment $(5,6)$. These protumor functions have been attributed to the M2 phenotype of TAMs. In contrast, the M1 phenotype of macrophages is known to sequester intracellular iron and induce an inflammatory response against tumor growth(6-8).

These opposing roles of macrophage phenotypes in tumor growth and response to treatment $(9,10)$, have been the basis for novel immunotherapeutic approaches aimed at promoting the anti-tumor M1 behavior $(11,12)$. However, in the absence of non-invasive biomarkers to monitor response to therapy, the assessment of the dominant TAM behavior in the tumor microenvironment requires invasive tissue sampling, which imposes a significant limitation to the advancement and real-time monitoring of novel immunotherapeutic approaches.

Currently, the radiological diagnosis of GBM and assessment of response to therapy are routinely performed through a battery of structural and physiological MRI modalities. Tumor progression is often associated with an increase in the size of contrast-enhanced areas or in the relative cerebral blood volume; however, these features still have significant limitations in the ability to differentiate response to treatment from tumor progression, particularly following administration of immunotherapeutic agents(13). Given the increasing knowledge about the role of immune microenvironment and the increasing number of immunotherapeutic trials for GBM, there is a great need for biomarkers that can non-invasively monitor the immune profile of the tumor microenvironment $(14,15)$.

MRI techniques that are sensitive to susceptibility such as gradient-echo-based relaxivity (R2*) measurement(16) and quantitative susceptibility mapping (QSM)(17) can provide valuable information on sources of magnetic field inhomogeneity, including iron concentration in the tissue(18-21). Considering the differential role of TAMs in iron metabolism $(6,7)$, the goal of this study was to assess the potential of MR susceptibility imaging as a non-invasive biomarker to predict TAM's dominant phenotype in the GBM microenvironment. In particular, we hypothesized that QSM and R2* measurements would correlate with TAM quantity and phenotype in GBM.

\section{Materials And Methods}

\section{SUBJECTS}

We screened subjects with intra-axial brain mass suggestive of high-grade glioma, who were referred to the radiology department of the Hospital of University of Pennsylvania from 2016 to 2019. Twenty-one adult patients who underwent tumor resection and whose tissue specimens were consistent with the histopathological diagnosis of GBM were enrolled in the study. 
This study was approved by the Institutional Review Board of the University of Pennsylvania, and all research was performed in accordance with relevant guidelines and regulations. Informed consent has been obtained from patients to include the additional research MRI sequence and histopathological studies as described in the following sections.

\section{Image Acquisition}

Imaging was performed on a 3 T scanner (Trio; Siemens) equipped with a 12-channel head coil. Single echo gradient echo MRI was obtained in the axial orientation using the following imaging parameters: inplane spatial resolution $=0.86 \times 0.86 \mathrm{~mm} 2$, slice thickness $=3 \mathrm{~mm}$, matrix $=256 \times 256 \times 24$, flip angle $(F A)=20, T E / T R=18 / 55 \mathrm{msec}$, and bandwidth $=444 \mathrm{~Hz} /$ pixel. Additional imaging included post-contrast T1 magnetization-prepared rapid gradient-echo (MPRAGE) (in-plane spatial resolution $=0.977 \times 0.977$ $\mathrm{mm} 2$, slice thickness $=1 \mathrm{~mm}$, matrix $=256 \times 192 \times 192, \mathrm{TE} / \mathrm{TR} / \mathrm{TI}=3.11 / 1750 / 950 \mathrm{msec}, \mathrm{FA}=15$, and bandwidth $=150 \mathrm{~Hz} /$ pixel) and fluid-attenuated inversion recovery imaging (FLAIR) (in-plane spatial resolution $=0.938 \times 0.938 \mathrm{~mm} 2$, slice thickness $=3 \mathrm{~mm}$, matrix $=256 \times 192 \times 60, \mathrm{TE} / \mathrm{TR} / \mathrm{TI}=$ $141 / 9420 / 2500 \mathrm{msec}, \mathrm{FA}=170$, and bandwidth $=287 \mathrm{~Hz} /$ pixel) after intravenous administration of gadolinium-containing contrast material (MultiHance, Bracco, Princeton, NJ or Dotarem, Guerbet); 0.1 $\mathrm{mmol} / \mathrm{kg}$, double dose. DSC-MR imaging was performed by a gradient-echo echo-planar (GE-EPI) imaging sequence during a second $0.1-\mathrm{mmol} / \mathrm{kg}$ bolus of contrast with the following parameters: TR/TE = $2000 / 45 \mathrm{~ms}, \mathrm{FOV}=22 \times 22 \mathrm{~cm} 2$, resolution $=1.72 \times 1.72 \times 3 \mathrm{~mm} 3,20$ sections.

For ex vivo imaging, we selected three subjects whose specimens had the least hemorrhage in order to minimize artifact in susceptibility maps on ultra-high field MRI. The formalin-fixed paraffin-embedded tissue samples for these subjects were imaged on a Bruker 9.4 Tesla $8.9 \mathrm{~cm}$ vertical bore MR as explained in detail in the supplementary material (see supplementary figure 1). T2 TurboRARE, and 3D multi-echo GRE scans were acquired at $60^{\circ} \mathrm{C}$ to ensure paraffin melt (See supplementary material and methods).

\section{Histology.}

A board-certified neuropathologist (M.P.N.), who was blinded to MRI susceptibility measurements, assessed the specimens from the initial surgical resections. Nineteen subjects had enough tissue for histological studies. Samples were formalin-fixed and paraffin-embedded, and the block that best represented the submitted tissue (often greater than $50 \%$ of the entire tissue) was chosen for additional staining.

Each specimen was stained with hematoxylin and eosin, as well as with CD68, CD86, CD206, and LFerritin. The unstained slides underwent heat-induced epitope retrieval in citrate buffer pH 6.0 (Leica Microsystems) for $20 \mathrm{~min}$. We then performed immunohistochemical staining on the Bond 111 Autostainer with the hematoxylin counterstain and DAB chromogen. 
For all subjects, histology slides were digitized at the Children Hospital of Philadelphia pathology core facility at $20 \mathrm{X}$ using an Aperio CS-O slide scanner (Leica Biosystems, Buffalo Grove, IL). These scanned images were preprocessed with QuPath software (22), using an automatic module to adjust the IHC stain vectors and remove unexpected colors due to artifacts. Areas of artifact (e.g. tissue folds, edge artifacts and scanning artifacts) were removed from the analysis through manual tissue segmentation using the QuPath annotations tool (22). For each case, the positivity percentage of the IHC images (i.e. CD68, CD86, CD206, and FL chain) was then quantified for the whole slide based on a threshold applied to the 3,3' Diaminobenzidine (DAB) signal in the cytoplasm. Refer to the supplementary material table-1 for the parameters used in positive cell detection. To address variation observed in staining intensity, for each IHC image, positive cell quantification was first performed using an initial threshold equal to mean $+2 S D$ of DAB optic density of all cells. To minimize the error rate in positive cell detection, this threshold was then modified by a neuropathologist (M.P.N) accordingly.

\section{Radiologic image analysis:}

All sequences were registered to post-contrast T1. using the FSL MRI toolkit. Regions of abnormal contrast enhancement, necrosis, and non-enhancing FLAIR signal intensity were segmented using a semiautomated segmentation tool (ITK-SNAP)(23) followed by manual editing by two board-certified neuroradiologists (S.A.N. and J.B.W.). Areas of susceptibility artifact on source DSC (dynamic susceptibility contrast) and areas with high signal intensity on pre-contrast T1 source were excluded from these segmentations as areas of hemorrhage.

QSM images were reconstructed from single echo gradient-echo MRI magnitude and phase data using the morphology-enabled dipole inversion (MEDI) algorithm(24), implemented in MATLAB (MathWorks, Natick, Massachusetts). A brain mask was obtained from the magnitude image using the FSL brain extraction tool (25). Subsequently, phase unwrapping was performed using Laplacian unwrapping (26), transmit phase was removed by fitting and subtracting a fourth-order 3D polynomial(27), and background field removal was performed using the regularization-enabled sophisticated harmonic artifact reduction for phase data (RESHARP) algorithm(28), prior to susceptibility inversion with MEDI using the default regularization parameters.

A threshold of 0.055 was applied to QSM values to exclude areas of artifact and venous structures. The thresholded QSM maps were reviewed and confirmed by a board-certified neuroradiologist (S.A.N.), who was blind to the immunohistochemistry results. The above-mentioned segmentations were applied to the QSM images and the voxel count and the average susceptibility of the segmented regions were recorded for each subject.

The T2* maps for the two ex-vivo samples were generated by a pixel-wise monoexponential fitting of the multi-echo GRE images using MATLAB (29).

\section{Pathology-radiology region of interest (ROI) analysis:}


For the cases with ex vivo MRI, we undertook an ROI approach for direct correlation analysis between the corresponding ROIs on IHC and MRI images. First, areas of highly dense tumor were determined by a tissue classification model (1 $\mu \mathrm{m} /$ pixel; random trees; QuPath 2.0.1)(22) that was trained by a neuropathologist (M.P.N) on H\&E images (figure 2). Subsequently, 16 ROls $\left(0.25 \mathrm{~mm}^{2}\right.$ each) were randomly placed in areas of tumor. The number of ROls per slide was proportional to the total area of tumor dense regions on each slide. For each ROI on the corresponding IHC images, the total number of positive cells was counted as described above. To transfer ROls to the corresponding coordinates on the T2* maps, IHC images were co-registered with MRI images using HistoloZee software (see Figure 3 ). The mean $T 2 *$ values $(19.5 \pm 11.7)(23)$ were then used to calculate the effective transverse relaxivity $\left(R 2^{*}=\right.$ $\left.1 / \mathrm{T} 2^{*}\right)$ for each ROI.

\section{Statistical Analysis}

We performed Shapiro -Wilk test to assess normality of data. For the correlation analyses between MRI metrics and histology, we used Pearson's R for normally distributed data and Spearman's Rho for nonnormally distributed data. Mean and standard deviation was reported for descriptive statistics. $P$ values below 0.05 were considered statistically significant. Statistical analysis was conducted in the R statistical environment (version 3.6.1; http://www.r-project.org/).

\section{Results}

\section{Subjects}

We studied 21 (mean age: $64.1+/-9.86$ years; $13 \mathrm{M}$; $8 \mathrm{~F}$ ) patients with treatment naïve glioblastoma who underwent tumor resection. Two patients were IDH-mutant and the remaining patients were IDH wild-type. Among the 19 subjects with in vivo MRI, one subject was excluded because of distortion of MRI data due to significant motion artifact. Also, among the three subjects that were selected for ex-vivo imaging, one was removed due to tissue distortion during MRI imaging. One subject underwent both in-vivo and ex vivo MRI imaging of the tumor specimen.

\section{In vivo MRI}

The analysis of in vivo subjects revealed a significant positive correlation between QSM-based mean susceptibility $(0.0044 \pm 0.0055)$ for the tumor enhancing zone and the L-ferritin positivity percent $(7.8 \pm$ $2.3 \%)$ in the entire specimen area $(r=0.56, p=0.018$; figure 1$)$. Moreover, the mean susceptibility significantly correlated with positivity percent on the whole slides for $\operatorname{CD} 68(9.8 \pm 4.2 \% ; \rho=0.52, p=0.034)$ and $\operatorname{CD} 86$ (11.95 $\pm 6.3 \% ; r=0.7 p=0.001)$, but not for $\operatorname{CD} 206(4.4 \pm 1.9 \% ; \rho=0.09 ; p=0.7)$ (figure 1).

In addition, mean susceptibility from the combination of tumor's enhancing zone and necrotic core correlated with the corresponding L-Ferritin $(r=0.72 ; p=0.001)$ and CD86 $(r=0.63 ; p=0.005)$ positivity percent for the entire specimen area, and demonstrated a trend toward significant correlation with CD68 $(r=0.46 ; p=0.06)$. 


\section{Ex vivo MRI}

A total of 16 ROls were randomly placed on the tumor dense areas of IHC slides from the two blocks that had ex vivo MRI. We observed a significant positive correlation between mean R2* values $(68.1 \pm 37.1)$ and CD68 positive cell counts for the corresponding ROIs ( $r=0.6, p=0.016$; figure 2$)$. Similarly, mean R2* values significantly correlated with CD86 $(r=0.54, p=0.03)$ but not with CD206 positive cell counts $(r=0.15$, $\mathrm{p}=0.5$; figure 2). Of note, we did not find a significant correlation between $\mathrm{R} 2 *$ measurements and $\mathrm{L}$ ferritin positive cells $(r=0.09 ; p=0.7)$.

\section{Discussion}

In this study, we examined the role of MRI susceptibility biomarkers, QSM and R2*, to determine the distribution of TAMs in GBM microenvironment based on their differential role in iron metabolism using both in-vivo results from clinical MRI and the proof of concept ex-vivo MR experiment. We observed a significant positive correlation between in vivo QSM-based susceptibility and ferritin light chain. Previous studies have shown that the distribution of ferritin and iron closely overlap in the brain(16). While Hferritin predominates in neurons $(16,30)$, in line with their high iron uptake and peroxidase activity, Lferritin is predominant in macrophages and microglia favoring their role in long-term iron storage $(16,31)$. Hence, our findings indicated that QSM measurements could predict iron storage in tumor-associated macrophages. We also observed that macrophage counts (as identified by general CD68 marker) significantly correlated with in vivo QSM, with higher susceptibility associated with higher CD68 cell counts. Of note, these results were replicated with CD 86 positive cells, representing an M1 phenotype; but not with CD206 positive cells, representing M2 phenotype. This finding is in line with the role of M1 phenotype in storing intracellular iron, and $\mathrm{M} 2$ phenotype in releasing iron in the tumor microenvironment(7). The M1 phenotype, is known to exert anti-tumor functions not only by triggering the inflammatory response but also by sequestering iron, on which tumor cells depend for their growth and proliferation $(6,8)$. This is in contrast to $M 2$ phenotype with anti-inflammatory functions that release iron into the tumor microenvironment, promoting tumor growth and proliferation $(6,8,12)$.

In this study, we also performed an ex vivo MR imaging on paraffin-embedded blocks from three subjects with minimal hemorrhage to allow comprehensive radiology-pathology correlation(32). Using a similar T2* relaxometry technique, several studies in preclinical tumor models demonstrated successful mapping of TAMs in treatment naïve tumors and in the context of immunotherapy $(19,20,32,33)$. We also observed that R2* values correlated with both CD68 and CD86 positive cell counts in multiple ROls randomly placed on areas of highly dense tumor and co-registered with MRI. This direct histology and MRI correlation provided a proof of concept for the application of MR susceptibility imaging in predicting the distribution of TAMs in human GBM microenvironment.

Given their role in tumor growth and response to treatment, TAMs have been the focus of recent research for the development of imaging biomarkers to assess prognosis as well as response to treatment in GBM $(34,35)$. Multiple studies involved macrophage modulation in tandem with systematic treatment for 
GBM, for example, in vivo models have shown that anti-CD47 treatment has the ability to differentiate macrophages into phenotypes the M1 phenotype(36), thereby inducing anti-tumor effects (37)and modulating growth. Furthermore, processes such as CD40 activation(38), Toll-like receptors (TLR) agonists(39), and TAM depletion(40,41), have all been investigated in targeting the recruitment of macrophages and therefore modulating tumor progression. Monitoring changes in iron content have also been shown to be beneficial to GBM treatment. Shenoy et. al., found that iron-loaded macrophages resisted conversion from inflammatory to immunosuppressive polarity, thus indicating the integral role of iron quantification in GBM treatment and prognosis(42). The results of their study further indicate the need to quantify the link between iron status and immune function for improving therapies for GBM.

A variety of MRI and PET imaging techniques have been used to quantify TAMs in gliomas (43), for example, in a pilot study of 10 adult subjects with GBM who underwent ferumoxytol-enhanced MRI, measurements of susceptibility obtained after ferumoxytol administration correlate with iron-containing macrophage concentration(34); however, this approach requires slow intravenous administration of ferumoxytol followed by next day delayed imaging which may limit the widespread use. Translocator protein (TSPO) specific PET radiotracers have also been used to monitors TAMs; however, the two main limitations are lack of phenotype (M1 vs. M2) specificity(43), and over-expression in tumor cells(44). To the best of our knowledge, our study is the first to provide both in vivo and ex vivo evidence for the application of non-invasive MRI imaging for TAMs quantification and spatial mapping in human GBM.

Our study had several limitations. Most importantly, we had a small sample size for in vivo and especially the ex vivo experiments given that most of the subjects were alive at the time of this study and we limited the ex-vivo experiments to subjects with a large amount of tumor tissue to avoid exhaustion of the specimen. Furthermore, one of our three ex-vivo samples was excluded from the study due to tissue distortion during the MRI procedure. Hence, to perform a correlation analysis between MRI and IHC positive cell counts on the remaining two samples, we used a random ROI placement approach. While the small sample size was a limiting factor, the direct spatial co-registration of the two modalities (45) provided proof of concept for the application of MRI susceptibility imaging in the prediction of TAMs' distribution. Finally, the presence of intratumoral hemorrhage may affect quantitative susceptibility mapping and T2* relaxometry; however, we did use source images of DSC perfusion sequence and other sequences to exclude areas of intratumoral susceptibility that were felt to reflect hemorrhage and the same methodology can be used to identify and exclude areas of hemorrhage from QSM maps in the clinical setting. In addition, areas of hemorrhage were excluded from tumor segmentation in ex vivo experiments.

In conclusion, we demonstrated encouraging evidence that MR susceptibility Imaging can quantify the iron content of GBM and provide a non-invasive method for TAM quantification and phenotyping, though further studies with larger samples size are needed to corroborate these results. We envision future applications of this research will include monitoring changes in iron content and macrophage composition during conventional GBM treatment regimens, as well as TAM modulating clinical trials, such as those described previously, to monitor treatment success and predict outcomes. 


\section{Declarations}

\section{Compliance with Ethical Standards:}

\section{Conflict of interest:}

The authors declare no potential conflicts of interest

Ethical approval: All procedures performed in studies involving human participants were in accordance with the ethical standards of the institutional research committee and with the 1964 Helsinki declaration and its later amendments.

\section{Informed consent:}

Informed consent was obtained from all individual participants included in the study.

\section{Authors contribution:}

Conceptualization: MacLean Nasrallah, Ali Nabavizadeh, Amir Nazem, Samantha C. Guiry, Yi Fan, Jeffrey B. Ware, Walter R. Witschey, Rahim Rizi, Stephen J. Bagley, Arati Desai, Donald M. O’Rourke, Steven Brem

Acquisition of data: Amir Nazem MD, Samantha C. Guiry, Mehrdad Pourfathi, Jeffrey B. Ware

Preprocessing of images: Amir Nazem, Samantha C. Guiry

Writing - original draft preparation: Amir Nazem, Samantha C. Guiry, Ali Nabavizadeh

Writing - review and editing: Amir Nazem MD, Samantha C. Guiry, Mehrdad Pourfathi, Jeffrey B. Ware, Hannah Anderson, Srikant Kamesh lyer, Brianna F Moon, Yi Fan, Walter R. Witschey, Rahim Rizi, Stephen J. Bagley, Arati Desai, Donald M. O’Rourke, Steven Brem, MacLean Nasrallah, Ali Nabavizadeh

\section{Data availability:}

The datasets generated during and/or analysed during the current study are available from the corresponding author on reasonable request.

\section{Study Funding:}

McCabe Fund Award granted to S.A.N from the Perelman School of Medicine, University of Pennsylvania

\section{References}

1. Thakkar JP, Dolecek TA, Horbinski C, Ostrom QT, Lightner DD, Barnholtz-Sloan JS et al (2014) Epidemiologic and molecular prognostic review of glioblastoma. Cancer epidemiology, biomarkers \& 
prevention: a publication of the American Association for Cancer Research, cosponsored by the American Society of Preventive Oncology. 23:1985-199610

2. Pombo Antunes AR, Scheyltjens I, Duerinck J, Neyns B, Movahedi K, Van Ginderachter JA (2020) Understanding the glioblastoma immune microenvironment as basis for the development of new immunotherapeutic strategies.eLife. ; 9

3. Chen Z, Hambardzumyan D (2018) Immune Microenvironment in Glioblastoma Subtypes. Front Immunol 9:1004

4. Sampson JH, Gunn MD, Fecci PE, Ashley DM (2020) Brain immunology and immunotherapy in brain tumours. Nature Research, pp 12-25

5. Morisse MC, Jouannet S, Dominguez-Villar M, Sanson M, Idbaih A (2018) Interactions between tumor-associated macrophages and tumor cells in glioblastoma: unraveling promising targeted therapies. Expert Rev Neurother 18(9):729-737

6. Pfeifhofer-Obermair C, Tymoszuk P, Petzer V, Weiss G, Nairz M (2018) Iron in the Tumor Microenvironment-Connecting the Dots. Front Oncol 8:549

7. Recalcati S, Locati M, Marini A, Santambrogio P, Zaninotto F, De Pizzol M et al (2010) Differential regulation of iron homeostasis during human macrophage polarized activation. Eur $\mathrm{J}$ Immunol 40(3):824-835

8. Sica A, Erreni M, Allavena P, Porta C (2015) Macrophage polarization in pathology. Birkhauser Verlag AG, pp 4111-4126

9. Leblond MM, Pérès EA, Helaine C, Gérault AN, Moulin D, Anfray C et al (2017) M2 macrophages are more resistant than $\mathrm{M} 1$ macrophages following radiation therapy in the context of glioblastoma. Oncotarget 8(42):72597-72612

10. Pollard JW (2004) Tumour-educated macrophages promote tumour progression and metastasis. European Association for Cardio-Thoracic Surgery, pp 71-78

11. Wang Q, He Z, Huang M, Liu T, Wang Y, Xu H et al (2018) Vascular niche IL-6 induces alternative macrophage activation in glioblastoma through HIF-2a. Nat Commun 9(1):559

12. Zhou K, Cheng T, Zhan J, Peng X, Zhang Y, Wen J et al (2020) Targeting tumor-associated macrophages in the tumor microenvironment. Oncology letters 20(5):234

13. Aquino D, Gioppo A, Finocchiaro G, Bruzzone MG, Cuccarini V (2017) MRI in Glioma Immunotherapy: Evidence, Pitfalls, and Perspectives. Journal of immunology research 2017:5813951

14. Klemm F, Maas RR, Bowman RL, Kornete M, Soukup K, Nassiri S et al (2020) Interrogation of the Microenvironmental Landscape in Brain Tumors Reveals Disease-Specific Alterations of Immune Cells. Cell 181(7):1643-60e17

15. Tan AC, Ashley DM, López GY, Malinzak M, Friedman HS, Khasraw M (2020) Management of glioblastoma: State of the art and future directions. Cancer J Clin 70(4):299-312

16. Haacke EM, Cheng NYC, House MJ, Liu Q, Neelavalli J, Ogg RJ et al (2005) Imaging iron stores in the brain using magnetic resonance imaging. Magn Reson Imaging 23(1):1-25 
17. Liu C, Li W, Tong KA, Yeom KW, Kuzminski S (2015) Susceptibility-weighted imaging and quantitative susceptibility mapping in the brain. John Wiley and Sons Inc., pp 23-41

18. Duyn J (2013) MR susceptibility imaging. J Magn Reson 229:198-207

19. Leftin A, Ben-Chetrit N, Klemm F, Joyce JA, Koutcher JA (2017) Iron imaging reveals tumor and metastasis macrophage hemosiderin deposits in breast cancer. PLoS ONE 12(9):e0184765

20. Leftin A, Zhao H, Turkekul M, de Stanchina E, Manova K, Koutcher JA (2017) Iron deposition is associated with differential macrophage infiltration and therapeutic response to iron chelation in prostate cancer. Sci Rep 7(1):11632

21. Schenck JF (1996) The role of magnetic susceptibility in magnetic resonance imaging: MRI magnetic compatibility of the first and second kinds. Med Phys 23(6):815-850

22. Bankhead P, Loughrey MB, Fernández JA, Dombrowski Y, McArt DG, Dunne PD et al (2017) QuPath: Open source software for digital pathology image analysis. Sci Rep 7(1):1-7

23. Yushkevich PA, Piven J, Hazlett HC, Smith RG, Ho S, Gee JC et al (2006) User-guided 3D active contour segmentation of anatomical structures: Significantly improved efficiency and reliability. Neurolmage 31(3):1116-1128

24. Liu T, Liu J, De Rochefort L, Spincemaille P, Khalidov I, Ledoux JR et al (2011) Morphology enabled dipole inversion (MEDI) from a single-angle acquisition: Comparison with COSMOS in human brain imaging. Magn Reson Med 66(3):777-783

25. Smith SM (2002) Fast robust automated brain extraction. Hum Brain Mapp 17(3):143-155

26. Bilgic B, Fan AP, Polimeni JR, Cauley SF, Bianciardi M, Adalsteinsson E et al (2014) Fast quantitative susceptibility mapping with L1-regularization and automatic parameter selection. Magn Reson Med 72(5):1444-1459

27. Langkammer C, Schweser F, Shmueli K, Kames C, Li X, Guo L et al (2018) Quantitative susceptibility mapping: Report from the 2016 reconstruction challenge. Magn Reson Med 79(3):1661-1673

28. Sun $\mathrm{H}$, Wilman $\mathrm{AH}$ (2014) Background field removal using spherical mean value filtering and Tikhonov regularization. Magn Reson Med 71(3):1151-7

29. Chavhan GB, Babyn PS, Thomas B, Shroff MM, Mark Haacke E (2009) Principles, techniques, and applications of T2*-based MR imaging and its special applications. Radiographics 29(5):1433-1449

30. Beard JL, Connor JR, Jones BC (1993) Iron in the Brain. Oxford Academic, pp 157-170

31. Connor JR, Menzies SL (1995) Cellular management of iron in the brain. J Neurol Sci 134(SUPPL):33-44

32. Matsuda KM, Lopes-Calcas A, Honke ML, O'Brien-Moran Z, Buist R, West M et al (2017) Ex vivo tissue imaging for radiology-pathology correlation: a pilot study with a small bore 7-T MRI in a rare pigmented ganglioglioma exhibiting complex MR signal characteristics associated with melanin and hemosiderin. Journal of medical imaging (Bellingham, Wash) 4(3):036001

33. Leftin A, Ben-Chetrit N, Joyce JA, Koutcher JA (2019) Imaging endogenous macrophage iron deposits reveals a metabolic biomarker of polarized tumor macrophage infiltration and response to 
CSF1R breast cancer immunotherapy. Sci Rep 9(1):857

34. Iv M, Samghabadi P, Holdsworth S, Gentles A, Rezaii P, Harsh G et al (2019) Quantification of Macrophages in High-Grade Gliomas by Using Ferumoxytol-enhanced MRI: A Pilot Study. Radiology 290(1):198-206

35. Zhou J, Reddy MV, Wilson BKJ, Blair DA, Taha A, Frampton CM et al (2018) MR Imaging Characteristics Associate with Tumor-Associated Macrophages in Glioblastoma and Provide an Improved Signature for Survival Prognostication. American Journal of Neuroradiology 39(2):252-9

36. Zhang M, Hutter G, Kahn SA, Azad TD, Gholamin S, Xu CY et al (2016) Anti-CD47 treatment stimulates phagocytosis of glioblastoma by $\mathrm{M} 1$ and $\mathrm{M} 2$ polarized macrophages and promotes $\mathrm{M} 1$ polarized macrophages in vivo.PLoS ONE. ; 11(4)

37. Li F, Lv B, Liu Y, Hua T, Han J, Sun C et al (2018) Blocking the CD47-SIRPa axis by delivery of antiCD47 antibody induces antitumor effects in glioma and glioma stem cells. Oncolmmunology. ; 7(2)

38. Hoves S, Ooi CH, Wolter C, Sade H, Bissinger S, Schmittnaegel M et al (2018) Rapid activation of tumor-associated macrophages boosts preexisting tumor immunity. J Exp Med 215(3):859-876

39. Urban-Wojciuk Z, Khan MM, Oyler BL, Fåhraeus R, Marek-Trzonkowska N, Nita-Lazar A et al (2019) The Role of TLRs in Anti-cancer Immunity and Tumor Rejection. Front Immunol 10:2388

40. Akkari L, Bowman RL, Tessier J, Klemm F, Handgraaf SM, de Groot M et al (2020) Dynamic changes in glioma macrophage populations after radiotherapy reveal CSF-1R inhibition as a strategy to overcome resistance.Science translational medicine. ; 12(552)

41. Pires-Afonso Y, Niclou SP, Michelucci A (2020) Revealing and Harnessing Tumour-Associated Microglia/Macrophage Heterogeneity in Glioblastoma.International journal of molecular sciences. ; 21(3)

42. Shenoy G, Madhankumar A, Slagle-Webb B, Mrowczynski O, Schell T, Nesterova D et al (2019) TMIC31. IMPACT OF IRON ON MACROPHAGE IMMUNE PHENOTYPE IN THE GLIOBLASTOMA TUMOR MICROENVIRONMENT. Neurooncology 21(Supplement6):vi254-vi

43. Mukherjee S, Sonanini D, Maurer A, Daldrup-Link HE (2019) The yin and yang of imaging tumor associated macrophages with PET and MRI. Theranostics 9(25):7730-7748

44. Ammer LM, Vollmann-Zwerenz A, Ruf V, Wetzel CH, Riemenschneider MJ, Albert NL et al (2020) The Role of Translocator Protein TSPO in Hallmarks of Glioblastoma.Cancers. ; 12(10)

45. Adler DH, Wisse LEM, Ittyerah R, Pluta JB, Ding SL, Xie L et al (2018) Characterizing the human hippocampus in aging and Alzheimer's disease using a computational atlas derived from ex vivo MRI and histology. Proc Natl Acad Sci USA 115(16):4252-7

\section{Figures}




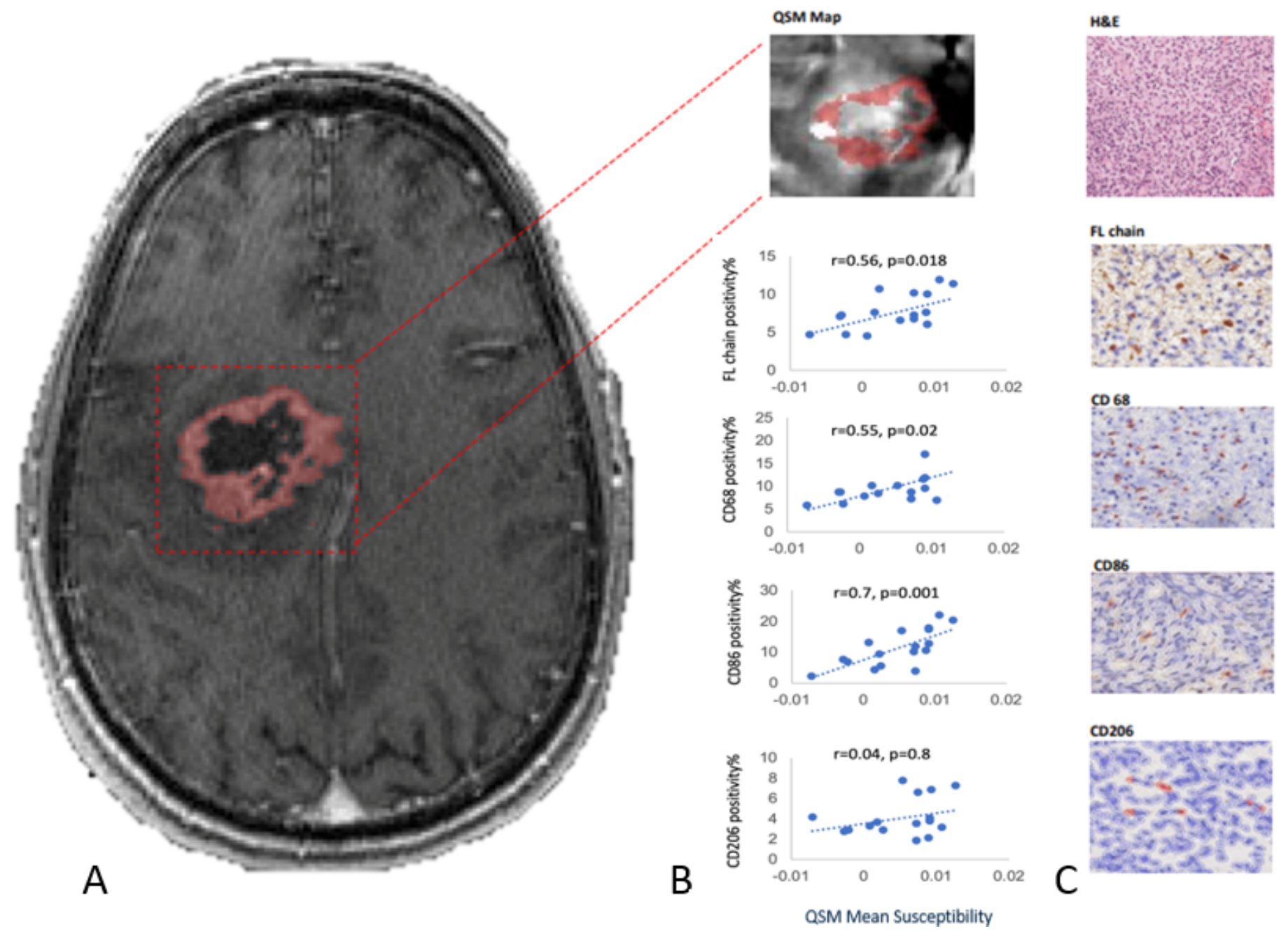

Figure 1

Quantitative susceptibility mapping of enhancing zone in GBM, and its relationship with ferritin light chain, CD68, CD86, and CD206 counts in tumor microenvironment. Tumor segmentation is shown in A. There was a significant positive correlation between QSM-based mean susceptibility for the tumor enhancing zone and the L-ferritin positivity percent. The mean susceptibility significantly correlated with positivity percent on the whole slides for CD68 and CD86, but not for CD206. 

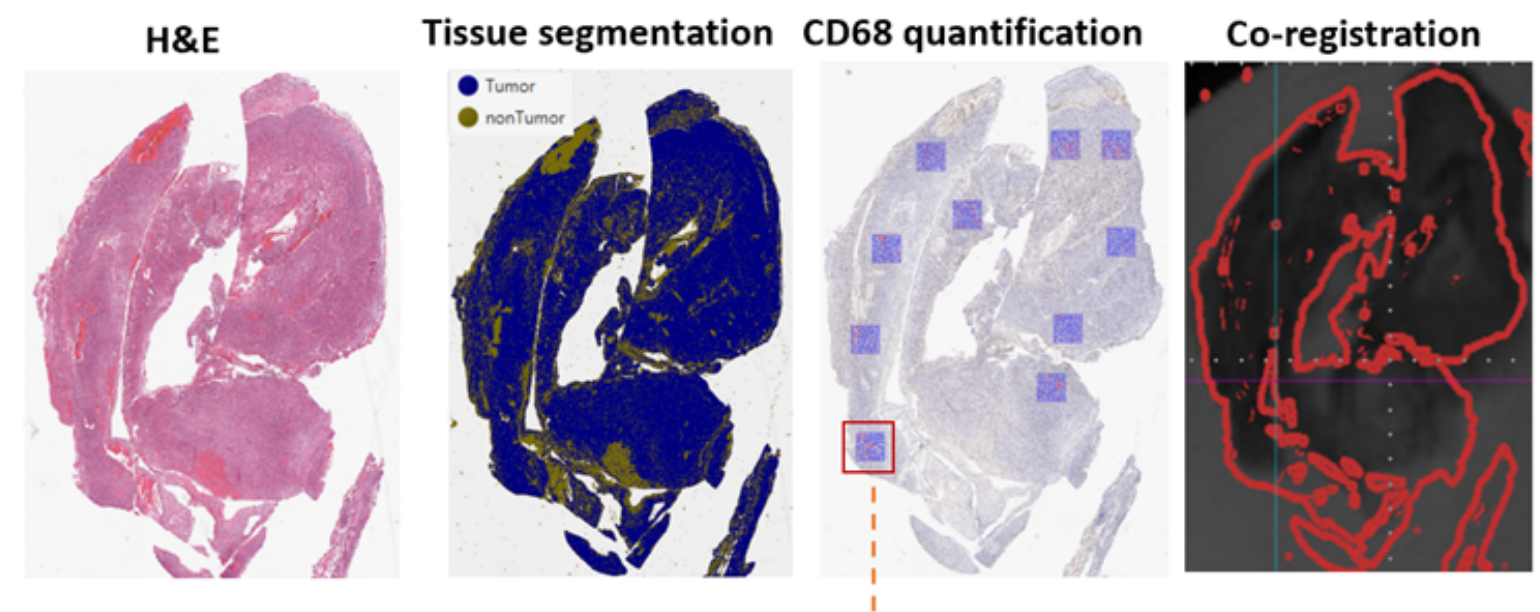

T2* ROI analysis
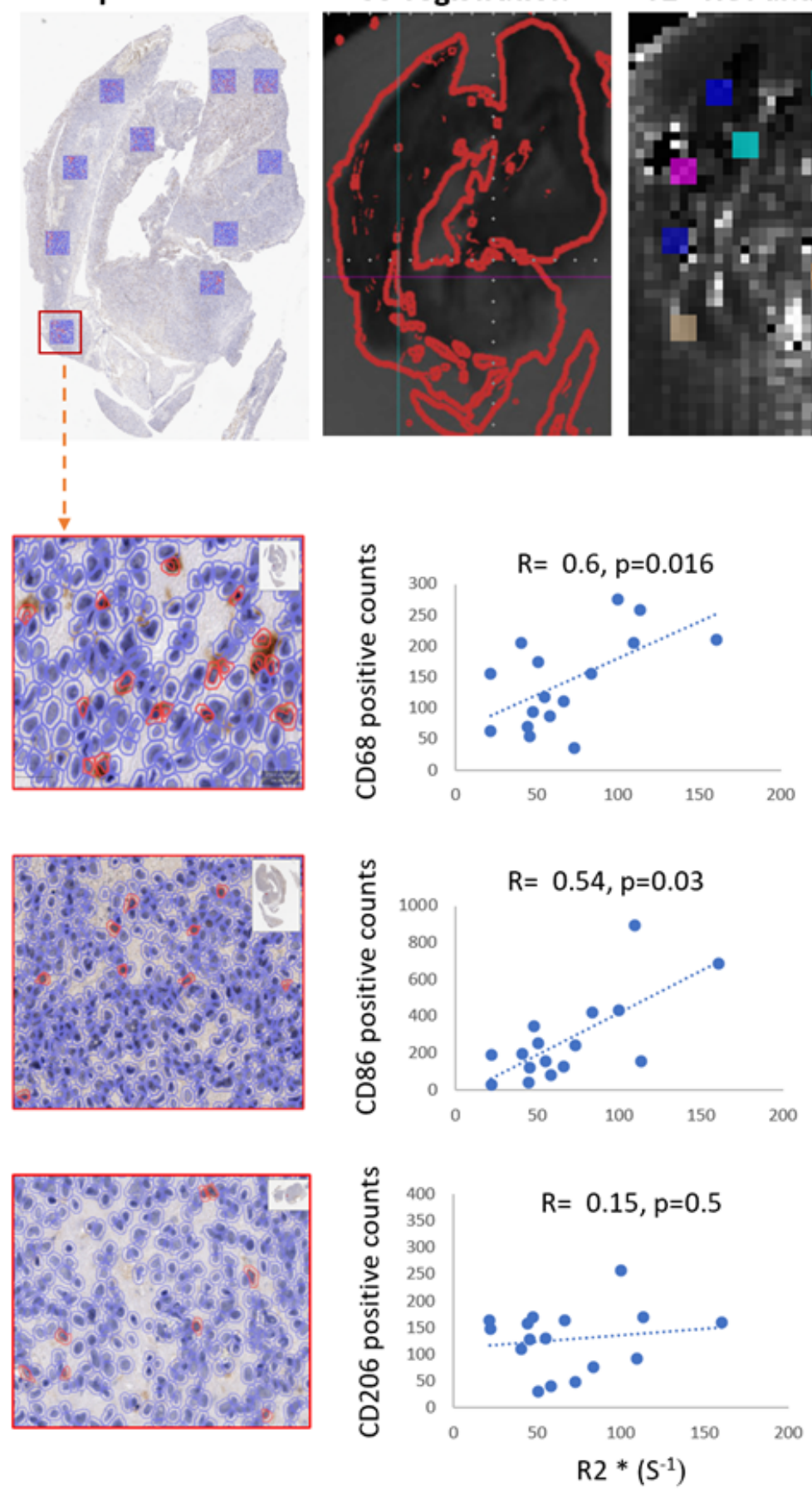

Figure 2

Ex vivo MRI and immunohistochemistry correlations. There is a statistically significant positive correlation between mean R2* values and CD 86 as well as CD 68 positive cell counts on corresponding ROIs $(n=16)$ but not with CD206 positive cell counts.

\section{Supplementary Files}


This is a list of supplementary files associated with this preprint. Click to download.

- SupplementaryMaterial.docx 